\title{
A MANAGEMENT FLIGHT SIMULATOR OF AN INTENSIVE CARE UNIT
}

\author{
Daniel Garcia-Vicuña \\ Fermin Mallor \\ Department of Statistic, IT and Mathematics \\ Public University of Navarre \\ Campus Arrosadia \\ Pamplona, 31006, SPAIN
}

\author{
Laida Esparza \\ Department of Critical Care Medicine \\ Navarre Hospital Compound \\ Irunlarrea-Str. 3 \\ Pamplona, 31008, SPAIN
}

\author{
Pedro Mateo \\ Department of Statistical Methods \\ University of Zaragoza \\ Pedro Cerbuna 12 \\ Zaragoza, 50009, SPAIN
}

\begin{abstract}
Management Flight Simulators (MFS) supply a simulated environment in which managers can learn from experience in a controlled setting. Although its use is usual in other areas, no such software has been developed to learn about the complexity of the Intensive Care Unit (ICU) management. This paper describes an MFS of ICUs which includes main features that distinguish it from other simulators such as the evolution of patients' health status and the recreation of real discharge and admission processes. The mathematical model is a discrete event simulation model in which different types of patients arrive at the ICU (emergency and scheduled patients). The user manages the simulated ICU by deciding about their admission or diversion and which inpatients are discharged. The analysis of recorded data is used to detect controversial scenarios and to understand how physicians' decisions are made.
\end{abstract}

\section{INTRODUCTION}

The Intensive Care Unit (ICU) is a hospital unit that is fundamental for the care of patients that combine high levels of severity with a reasonable expectation of recovery. In ICUs, it is possible to provide artificial support to more than one organ system at the same time (respiratory, circulatory, neurological, etc.). This, together with the aging of the population has led to an increase in the number of patients admitted in ICUs. However, the cost associated with these units is much higher than the costs attributed to the hospitalization in conventional beds, due to the high technology implemented and the high number of professionals that support these.

Therefore, bed management in an ICU is a problem with a double perspective. On the one hand, there is the high price of the service, caused mainly by the high fixed costs of the beds (independent of the level of occupancy). Thus, from an economic point of view, managers will seek to maximize the bed occupancy of the ICU to avoid its underutilization (Halpern and Pastores 2010). On the other hand, situations of lack of beds trigger several consequences such as the suspension of scheduled surgeries, the delay of admissions in the ICU and the early or inadequate discharge of a patient to free up his/her bed which are associated with poorer prognosis (Chalfin et al. 2007; Cardoso et al. 2011). Therefore, from a clinical point of view, high occupations will be avoided. 


\section{Garcia-Vicuña, Mallor, Esparza, and Mateo}

Teres (1993) posed as one of the great ethical dilemmas a situation that he called "the ritual of the last bed". The stochastic nature of both the arrival of patients at ICU, as well as the length of their stays, makes inevitable that periodically the occupation of the ICU is close to the limit. On a day-to-day basis, it is the medical staff of the ICU that must adjust the demand for beds with the service capacity and decide on the admission of new patients and the discharge time of the inpatients.

In addition to pressure for lack of beds, there are other factors that also influence when a patient is discharged, such as the variability between physicians when determining the stability of a patient. Although variability is not a negative aspect, inappropriate variation can lead to inefficiencies in the quality of care.

The analysis of patient-admission and inpatient-discharge decisions can be done safely in virtual environments that reproduce with high fidelity the characteristics and dynamics of an ICU. The aim of this paper is to present an interactive simulator that mimics a real ICU and records the discharge decisions made by users. The main features that distinguish this simulator from others are the simulation of the patient stay by evolving their health status (instead of using a single value for the length of stay (LoS)) and the recreation of real discharge and admission processes. Both elements are determinant for creating credible virtual scenarios allowing the users the management of the ICU as they would do in a real ICU, that is, with the same information and environment. The analysis of the recorded data about admission/discharge decisions and the statistics about cancelled surgeries, inpatients discharged in advance, admissions delayed and diverted patients, are used to characterize bed management policies implemented by users. Furthermore, differences between users can be detected and quantified as well as the identification of scenarios in which decisions differ the most. These controversial scenarios are of special interest for physicians because they support the discussion to elaborate consensus protocols for triage decisions in the hospital that can help to reduce variability in medical practice.

The rest of the paper is organized as follows: in Section 2 related literature is briefly reviewed, Section 3 presents the mathematical modelling of the ICU dynamics and its implementation in a discrete event simulation model (DES); the definition of virtual ICUs and recreation of different scenarios, as well as the interaction with the user and the recorded data are detailed in Section 4. We end the paper with a Section of conclusions.

\section{RELATED WORK}

In order to design a good mathematical model in the field of healthcare, it is necessary to account for both types of bibliography, medical and mathematical. Many mathematical models make assumptions that do not hold in the practice, which is the main reason for low success in the implementation of their results (Brailsford and Vissers 2011).

There is a broad medical literature describing the patient discharge decisions and reporting about their consequences. For instance, when a physician decides to shorten a patient's stay in order to admit another when there are no free beds, there is an increase in hospital mortality and patient LoS (Rodriguez-Carvajal et al. 2011; Ouanes et al. 2012), as well as in the risk of readmission to the ICU (Frost et al. 2010; Gantner et al. 2014). Some mathematical literature also discusses such discharge decisions, but usually they are not included in the mathematical models (Costa et al. 2003; Shmueli et al. 2003). Only a few mathematical models include this decision-making process (Dobson et al. 2010; Chan et al. 2012), but they lack some important elements (we refer to them in Section 3) that prevent them from analyzing real ICU management policies.

One of the classic problems addressed by simulation related to healthcare is ICU dimensioning and management optimization. ICUs are complex stochastic systems, so the simulation is a suitable tool to study them. Bai et al. (2018) offer a review of the use of operations research methods, including simulation, in ICU management. In order to obtain valid simulation models, it is necessary to include the process of physician's patient-discharge decision-making (Barado et al. 2012; Mallor and Azcárate 2014). It is certainly very difficult to create models for the decision making applied by physicians from the recorded data. In fact, many researchers approach this modelling problem from a normative point of view suggesting parameterized models that indicate how physicians' decisions should be made and then calibrating or 
optimizing the model parameters (Kim et al. 2000; Griffiths et al. 2013; Mallor et al. 2015; Mallor et al. 2016). There are also some studies (Ridge et al. 1998) in which early discharge is suggested as a bed management tool, but they are not explicitly modelled.

The literature reviewed shows that some researches can propose mathematical solutions to problems associated with ICU capacity and bed management. But the fact is that there are no articles found which provide analysis of how physicians' decisions are really made in ICUs. One way to collect this type of data is through management flight simulators (MFS), which supply a simulated environment in which managers can learn from experience in a controlled setting.

MFS, also known in the literature as virtual simulations, can be used both for research and as a learningtraining tool (Lamé and Simmons 2018). For research, this tool enables to induce decision-making processes, test theories about decision-making, analyze key processes and detect biases. No articles in which these methods are used in Healthcare to study physicians' decision-making have been found. But in other contexts such as economy (Sterman 1989) and environmental care (Moxnes and Saysel 2009), simulators have been used to analyze the behavior of a group of people.

Nevertheless, many Healthcare applications have been developed in which virtual simulation has a relevant role in order to teach students at universities and especially to learn from the experience of using. Most of those simulations use patient care manikins, only a few with computers or simulation models but none of them in ICU context. Grunewald et al. (2003) developed an interactive Web-based training program for radiology, which offers cases and exercises in radiographic anatomy at different selectable levels of difficulty. Rauner et al. (2016) provide both a training and a learning tool for mass casualty incidents. Bean et al. (2017) programmed a patient flow simulator with which professionals and students can learn important concepts about Healthcare management. After distributing resources between wards and deciding how those resources should be prioritized, users can observe how the flow of patients has been managed.

\section{MODELLING AN INTENSIVE CARE UNIT}

\subsection{Modelling the Patient Flow and Admission/Discharge Decisions}

An ICU can be mathematically modelled into the framework of queuing models. This mathematical scheme, followed by most of the ICU simulation models, is extended in this work by including new elements, such as discharge process times and patient health status, in order to provide a more realistic environment.

An ICU is represented by a queuing model by considering that servers are ICU beds, clients are patients that arrive according to a known schedule for those coming from elective surgeries or randomly for emergency patients, there is no waiting room and the queue discipline is "first come, first served". Finally, the service is provided individually, with duration dependent on the illness of each patient. This description leads to a queuing model $G / G / c / c$, where $c$ is the number of the beds in the ICU.

However, this model assumes admissions and discharges at any time and fails in modelling the dependence of the LoS of a patient with the congestion level of the ICU. According to physicians, these situations do not correspond to the real practice and in addition, the queuing model lacks the representation of admission and discharge processes. To overcome these drawbacks the basic $G / G / c / c$ queueing model is extended to include both processes as well as the modelling of the patient health status, which will allow the users of the interactive simulator to make informed decisions as physicians do in the real practice.

Discharge and admission process. Both the discharge and the admission of a patient are complex and not automatic processes. Discharging process would be delayed if there were no free beds in the ward to which the patient is being transferred. Moreover, families have to be committed to accompany and take care of the patient. Regarding the admission process, sometimes a setup time is required in order to clean or disinfect a recently freed up bed. Only when a patient arrives at ICU and there is a totally cleaned bed, the admission of the patient is virtually instantaneous. For this reason, most ICUs have a reserved room in which an admitted patient can temporarily be treated until a bed is completely freed up. Physicians locate 
patients there exceptionally and for a limited time, so this place is not like emergency waiting rooms where patients are queuing until being attended.

Figure 1 shows a diagram where both discharge and admission process are represented. On the right side, we can see a dark grey colored bed, which means that there is a patient waiting to be transferred because the discharge decision has just been made. The brown colored bed represents a deceased patient waiting for discharging too. When these two types of beds are freed up, the cleaning process starts (light grey color bed in Figure 1). After that, beds are available to be occupied with new admitted patients. On the left side, we can see both types of arrivals. Scheduled patients come from the operating room, and after that, if there is a bed available, they occupy it like when emergency admitted patients arrive. If not, both of them are temporarily located in those reserved beds (light blue color) until a bed has been completely freed up. The other three colors assigned to beds (red, orange and green) refer to three different categories of patient health status that are related to discharge decisions and that is explained in Section 4.3.

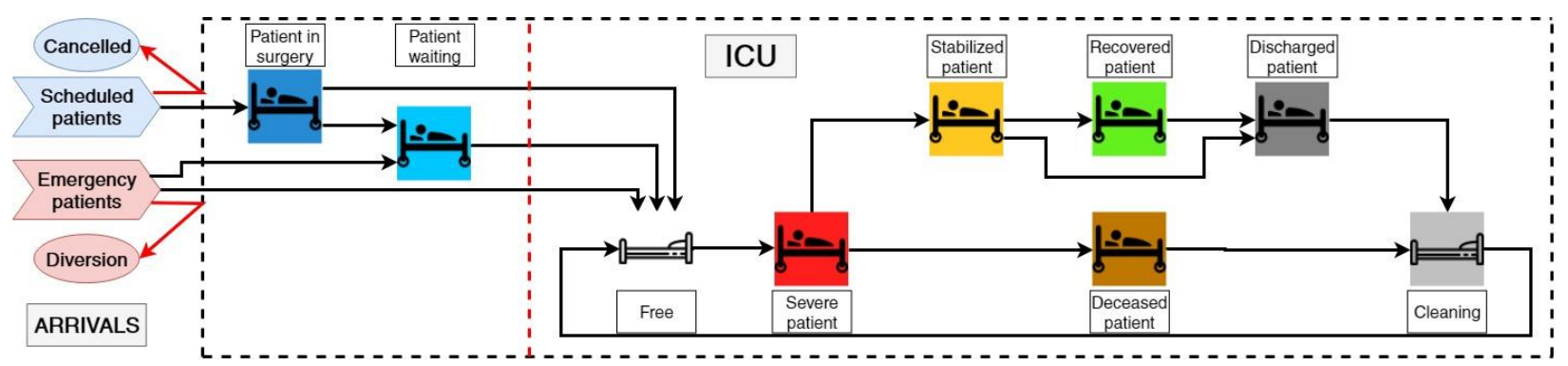

Figure 1: Representation of the dynamics of an ICU through the bed's state change diagram. The two types of patients are distinguished and also the direct entry to the ICU from a delayed one.

Discharge decision times. Decision making about the discharge of patients normally occurs only at a few scheduled times of the day. These moments are denominated clinical sessions and can take place once, twice or even three times a day (morning, afternoon and evening), depending on the ICU. During clinical sessions, physicians analyze discharge decisions considering ICU bed occupancy, patient health status and scheduled arrivals from surgery (described in Section 4.1). In the morning session, they also confirm or cancel surgeries of that day. When an emergency patient arrives at ICU, physicians decide on his/her admission. Only if there is no bed available, a patient discharge decision is required. In the case of discharge of patient and no rejection of the incoming one, the arriving patient can be exceptionally admitted and located in the reserved room as we have just explained.

Patient's health status. The queuing model represents the LoS of a patient as the realization of a certain random variable with a probability distribution fitted usually by using historical data. Therefore, this sampled time defines the event in which the patient is automatically discharged. However, the implementation of this approach in the simulator prevents from making decisions clinically grounded and informed. In this case, admission/discharge decision should rely on probability properties of the probability distribution used, as for example the expected remaining time. To overcome this strong drawback we model the health status of a patient by using 275 health indicators (medical and nursing reports included), all recorded by the software Metavision ${ }^{\circledR}$, which is a dedicated software to monitor the health of inpatients of ICU. These variables (described in Appendix A) give an extended and realistic description of the evolution of the patient health status, whose evolution can improve or get worse. Several patients get infections, even some of them die. In fact, they are used by physicians to assess the health condition of a patient in order to decide whether the patient is stable enough to be transferred to a lower level of care. These health indicators include neurological, hemodynamic, respiratory parameters among others such as provenance and principal diagnosis. 
All these processes and elements are included in a mathematical model that is organized as a Discrete Event Simulation (DES) Model, which is described in the next subsection.

\subsection{The Discrete Event Simulation Model}

A DES model is defined by the set of state variables, which provide at any time a complete description of the simulated system, and the set of events, which modify the value of state variables. Three different kinds of state variables were used to describe the ICU at any time: the first one describes the patients already accepted but not admitted in the ICU yet, the second category is composed by the 275 state variables describing the health status of each inpatient (some of them are qualitative prognosis of physicians and nurses) and the third one describes the occupancy state of each bed.

Each ICU's bed can be in one of the following states (represented by different colors in Figure 1):

- Free: the bed is completely available for the admission of a patient.

- With a deceased patient: there is a patient who has just died and is waiting for discharge.

- With a severe patient: there is a very serious patient who cannot be discharged under no circumstances.

- With a stabilized patient: there is a stabilized patient who could be considered eligible to be discharged under circumstances of high occupancy pressure.

- With a recovered patient: there is a patient who has recovered and is ready to be discharged.

- With a discharged patient: there is a patient who is waiting for transfer to a lower level of care. Discharge decision has already been made.

- Cleaning: the bed is undergoing cleaning tasks to condition it for the admission of a new patient.

Events that modify through time the value of state variables are grouped in four different categories:

- Patients arrival times (emergency or scheduled patients). There are two different groups of patients arriving at ICU: emergency patients and scheduled patients, each one with their own arrival pattern. Emergency patients arrivals occur 24/7 according to a Poisson Process (PP) with arrival rate $\lambda_{E}$. Each emergency patient is classified in one of the 6 illness groups (urgent surgery, polytrauma treated surgically or without intervention, patient hospitalized in Medical Service, patient hospitalized in Surgical Service, emergency/observation patient and patient admitted for organ donation/others) according to probabilities $P_{E i} i=1, \ldots, 6$ that define the mix of emergency patients. Once per week (on Fridays), the number of elective surgeries for each day of next week is simulated, according to the method explained in Section 4.1. These patients can be in the ICU for an expected short stay to recover from standard surgery procedure, with probability $P_{S 1}$, or for an expected long stay due to a complicated surgery or critical condition of the patient, with probability $P_{S 2}$. Values of different parameters are introduced in the simulator to define different ICU scenarios.

- Events producing changes in the value of the patient's clinical variables. We use historical data recorded by the Metavision ${ }^{\circledR}$ software to create the sequence of events in which each one of the health status variables changes of value. In this way, the simulation reproduces exactly health information of real ICU patients and their process of recovery (or worsening to death).

- Events that define discharge/admission decision times. At the times when clinical sessions are programmed the simulation stops waiting for the input of the user regarding discharge and admission decisions. The event of stopping the simulation waiting for the feedback of the user also happens in the case that an urgent patient arrives. In these moments, an admission decision must be made and exceptionally, when the ICU is fully occupied (or all free beds and in cleaning process beds are already assigned to patients that will be admitted shortly), a discharge decision too.

- Events that modify beds' condition. A bed changes its status when some of the previous events happen. For example, changes in patient clinical variables can produce that the patient transits to a 
stabilized health or to a recovery condition and then her/his bed does too. A discharge or an admission decision made at a decision time also changes the condition of the bed. Other events changing the state of a bed are the end of a discharge process and the end of a cleaning process.

\section{SIMULATOR}

\subsection{Defining Different Scenarios for ICUs}

We have designed and implemented computationally a dynamic simulator that generates scenarios like those found in a real ICU. The simulator is flexible enough to allow the definition of different ICUs according to its size, mix of patients, congestion level and discharge/admission decision process. Figure 2 shows how all these parameters can be modified. Moreover, it is possible to save all different scenarios that we create in that screen, to use directly in any simulation.

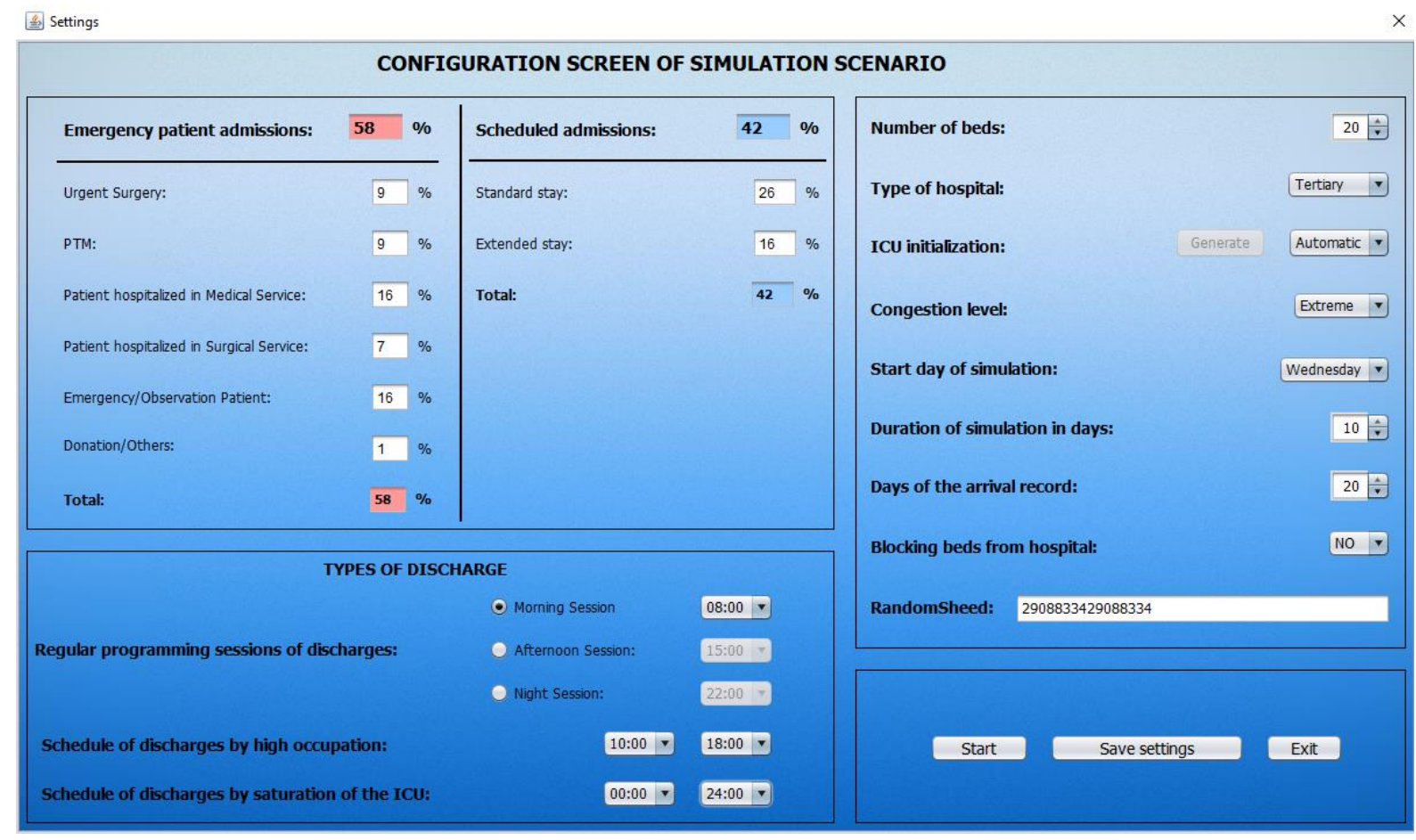

Figure 2: Configuration screen of simulation scenario.

The size is defined by the number of beds $c$. The mix of patients is defined by assigning a percentage for emergency and scheduled patients as we mentioned before, and as it is shown in equation (1).

$$
P_{E}+P_{S}=\sum_{i=1}^{6} P_{E_{i}}+\sum_{i=1}^{2} P_{S_{i}}=100 \%
$$

The health status of patients is simulated from 200 clinical reports of 200 real patients treated in ICU of Navarre Hospital Compound (112 emergency patients and 82 scheduled patients, distributed among their 6 and 2 categories, respectively), which have been completely anonymized.

The user can define the congestion level $\rho$ of the ICU and from it, it is possible to determine the patient arrival processes (for both programmed and emergency patients). The analysis of historical data allows to estimate the average time in ICU of each category of patient, $\operatorname{LoS}\left(E_{i}\right)$ and $\operatorname{LoS}\left(S_{i}\right)$, and therefore the total average $\operatorname{LoS}(1 / \mu)$ is estimated as 


$$
\frac{1}{\hat{\mu}}=\sum_{i=1}^{6} P_{E_{i}} \cdot \hat{\mathrm{E}}\left(\operatorname{LoS}\left(E_{i}\right)\right)+\sum_{i=1}^{2} P_{S_{i}} \cdot \hat{\mathrm{E}}\left(\operatorname{LoS}\left(S_{i}\right)\right) .
$$

The congestion level (high, very high or extreme) refers to the value of $\sigma=\lambda_{T} /(c \cdot \mu)$, where $\lambda_{T}$ is the total arrival rate. Once the value of $\sigma$ is set the value of $\lambda$ will be determined for each category of patients $\left(\lambda_{E}=P_{E} \cdot \lambda_{T}\right.$ and $\left.\lambda_{S}=P_{S} \cdot \lambda_{T}\right)$.

On the one hand, emergency patients present a PP, with arrival rate $\lambda_{E}$. When an emergency patient arrives at time $t_{i}$, the next patient will arrive at time $t_{i+1}$ obtained from the equation (2). Apart from the arrival time, we assign the type of this emergency patient who arrives. $\mathrm{He} / \mathrm{she}$ will be a patient of type $E_{i}$ with a probability of $P_{E i} / P_{E}$.

$$
t_{i+1}=t_{i}-\left(\frac{1}{\lambda_{E}}\right) \cdot \ln u_{i} ; \text { with } u_{i} \leadsto U(0,1)
$$

On the other hand, scheduled patients are shown to physicians at the time of the first clinical session in the morning. Those patients who are admitted arrive at ICU when the surgery is finished (each scheduled patient has this time previously defined). We consider that there are only operation days (OD) from Monday to Friday $(d=5)$; therefore, the expected number of surgeries a surgery day is $\lambda_{S *}=\lambda_{S} \cdot 7 / d$. For each type of scheduled patients the expected number of arrivals is $\lambda_{S * i}=\lambda_{S *} \cdot P_{S i} / P_{S}$. From these expected values the number of arrivals of each type of patient $S_{i}$ in each day are simulated as $\left[\lambda_{S * i}\right\rfloor$ patients with probability $\left\lfloor\lambda_{S * i}\right\rfloor+1-\lambda_{S * i}$, and $\left\lfloor\lambda_{S * i}\right\rfloor+1$ with probability $\lambda_{S * i}-\left\lfloor\lambda_{S * i}\right\rfloor$ (where $[$.$\rfloor denotes the integer$ part of the number).

Finally, the discharge/admission decision process is defined in the lower left side of Figure 2. It is possible to set different timetable of clinical sessions, as well as schedules of discharges by high occupation or saturation of the ICU (moments in which physicians can assign a discharge in order to reduce the occupation of the ICU, with a view to possible future admission or when a patient must be admitted obligatorily, but there are no free beds respectively). Out of hours defined there, discharges cannot be assigned.

\subsection{Initialization Process}

The initialization process of the ICU simulation, once the size $c$, congestion $\rho$ and mix of patients are defined, generates the number of inpatients at the beginning of the simulation, as well as their type and their health status.

We denote by $G_{i} i=1, \ldots, 8$ the eight groups of patients $E_{i} i=1, \ldots, 6$ and $S_{i} i=1,2$, each one with a LoS described by a random variable $T_{i}$. Let $\tau_{i}$ be the expected LoS of group $G_{i}\left(\tau_{i}=E\left(T_{i}\right)\right.$ ). The number of occupied beds is determined as a rounded value of the expected one, that is, $\lfloor\rho c\rfloor$ occupied beds with probability $\lfloor\rho c\rfloor+1-\rho c$, and $\lfloor\rho c\rfloor+1$ with probability $\rho c-\lfloor\rho c\rfloor$.

The probability that a patient of certain type occupies a bed is calculated as the percentage of time that a bed is occupied for that type of patient; that is, $\theta_{i}=\lambda_{i} \tau_{i} / \sum_{j} \lambda_{j} \tau_{j}$. Once the group of patients $i$ is selected, the specific patient $j$ that is going to occupy the bed is selected according to a probability that is proportional to the $\operatorname{LoS}$, that is, $\varphi_{i j}=t_{i j} / \sum_{k} t_{i k}$, where $t_{i k}$ is the $\operatorname{LoS}$ of the $k t h$ patient of the group $i$.

We need to start the simulation of the ICU representing its stationary state. For this reason, once a patient is selected to occupy a bed, the LoS already consumed at the beginning of simulation is considered uniformly distributed in his/her total LoS. Then, the health status of the patient is fast-forwarded according to his/her remaining LoS.

\subsection{Interaction with the User}

The simulator will be interactively managed by the user. At decision times, the simulator presents the user the patients who are occupying the ICU and he/she must make decisions like those made by physicians in 
the ICU, by analyzing the inpatient's health status, the occupancy level, and the forecasted scheduled patients.

Figure 3 shows the main screen of the simulator during the simulation. In the lower part, there is a history of emergency patients arrivals (left) and of the surgeries scheduled for the next few days (right). On the top right side, it is shown the events related to admissions, discharges or changes in the health status of patients. In the upper left part, there is a graphic panel in which the occupation of the ICU is represented. Clicking on a patient, you get all the clinical history to date (the evolution of the 275 variables describing the health status as well as the medical and nursery reports). In fact, the health status is reported in a different screen of the simulator that imitates and provides the same information that is displayed and recorded by the dedicated software Metavision ${ }^{\circledR}$. In this way, the simulator creates a totally realistic and credible ICU environment (see Figure 4).

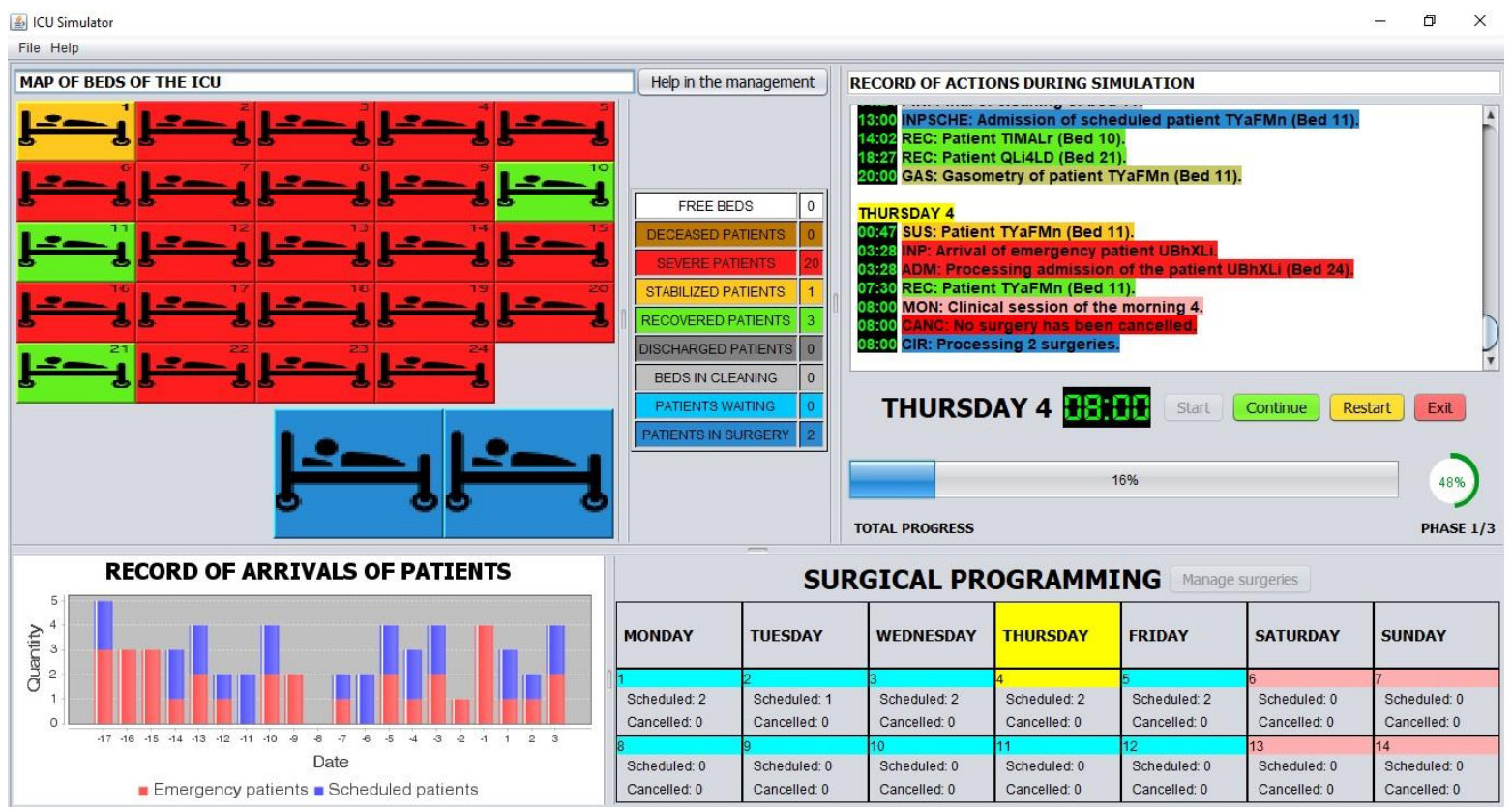

Figure 3: Simulator's main screen.

As we mentioned before, we have collected variables of 200 real patients of an ICU. The purpose of the simulator is not to evaluate the user's medical knowledge, but to collect information about his/her management. Therefore, the physician author of this paper together with her colleagues from the ICU examined all patients' clinical histories to define three states along their LoS, which help the user to make decisions. A patient who has just been admitted in the ICU is considered in severe conditions to be discharged (red color in Figure 1). In the other hand, patients at the end of their LoS are considered totally recovered and they should be discharged (green color in Figure 1). Finally, an intermediate state is established for each patient (orange color in Figure 1), which indicates the time from which the patient is sufficiently stabilized to be discharged, although risks to his/her health are assumed.

At decision moments (clinical sessions and at patient arrival times) the simulator waits for the decision of the user. He/She must decide which patients are discharged and which are not. In turn, the user of the simulator can decide if a patient's admission (emergency patient or scheduled patient) is denied due to lack of beds, or if an early discharge is assigned to a patient. When simulation finishes, all these decisions and general results (number of patients admitted, number of surgeries cancelled, number of early discharges...) are recorded in order to be analyzed after. Since a well-done simulation run could last many minutes the simulator allows the user to save the simulation and continuing it later. 


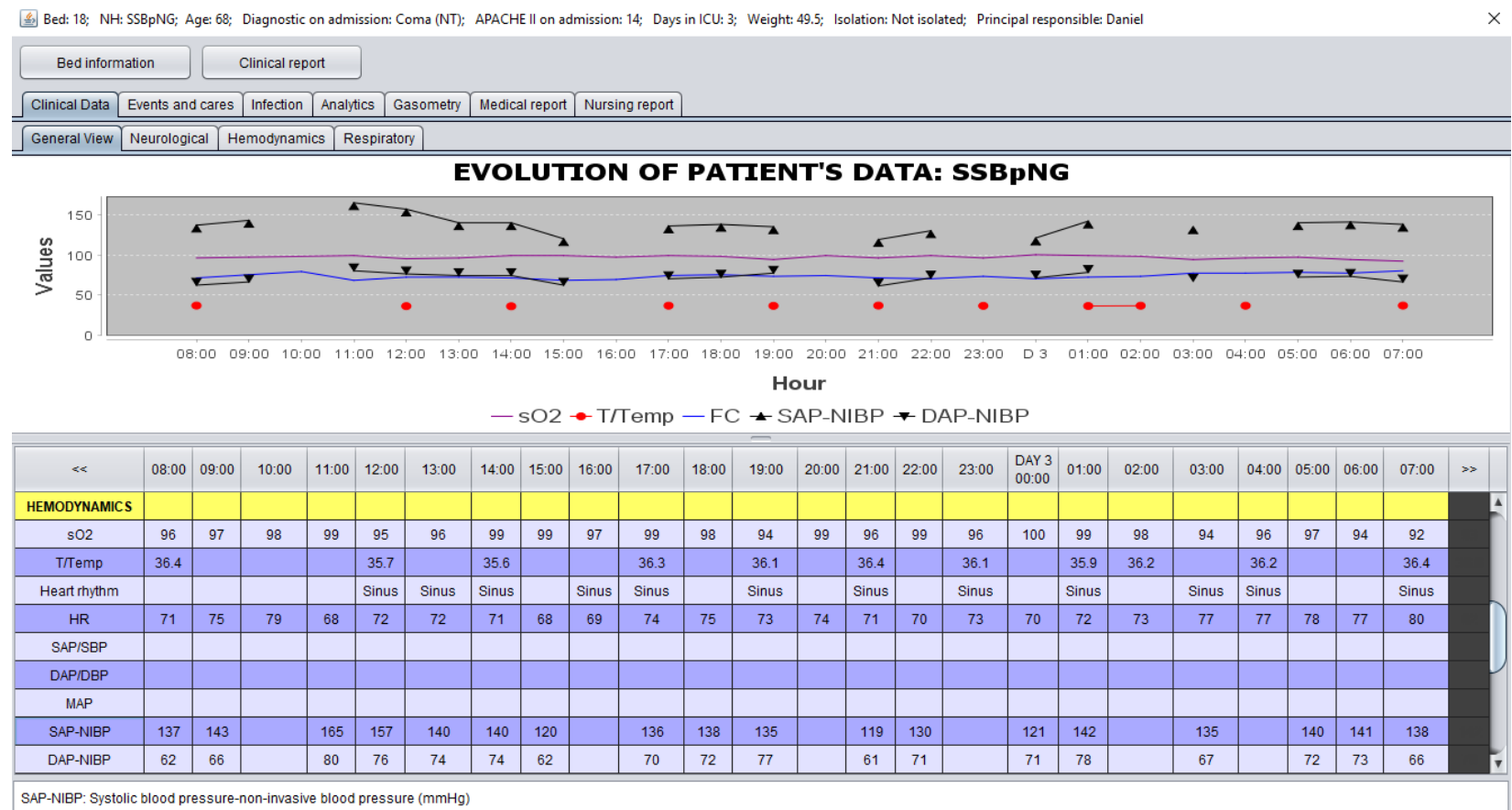

Figure 4: Information of patient's clinical data, mimicking real screens of Metavision®.

\subsection{Information Recorded}

This simulator will allow us to study decision-making in ICUs by requesting inputs from physicians. After each simulation, all decisions will be recorded in a file which can be opened by the simulator. These files allow reproducing step by step one simulation that is already finished. This is very useful in order to watch how simulation has been performed by another user, that could be a senior physician and used as a learning tool.

The recorded information can be used to analyze the management style of the user. For instance, Table 1 summarizes results recorded while we were testing the simulator with physicians. These preliminary results show general measures which allow us to compare the management of different users under exactly the same ICU scenario (that means the same initial inpatients and the same patient arrival process).

Table 1: Comparison of simulation global results recorded while some physicians were testing the simulator. Users faced a 15-bed ICU, which was initialized with 14 patients in different health statuses. Over ten days, 20 emergency patients and 14 scheduled patients arrived at the ICU.

\begin{tabular}{ccccl}
\hline Physician & $\begin{array}{c}\text { Diverted } \\
\text { emergency } \\
\text { patients }\end{array}$ & $\begin{array}{c}\text { Cancelled } \\
\text { surgeries }\end{array}$ & $\begin{array}{c}\text { Shortened } \\
\text { stays }\end{array}$ & $\begin{array}{c}\text { Average time of } \\
\text { shortened LoS (h) }\end{array}$ \\
\hline User 1 & 9 & 0 & 8 & 35.403 \\
User 2 & 10 & 2 & 10 & 37.95 \\
User 3 & 5 & 6 & 7 & 41.157 \\
User 4 & 8 & 1 & 12 & 37.879 \\
User 5 & 9 & 5 & 6 & 23.968 \\
User 6 & 8 & 6 & 2 & 47.5 \\
Mean & 8.167 & 3.333 & 7.5 & 37.301 \\
\hline
\end{tabular}


The simulator has been used and validated by ICU physicians of the main hospital in Navarre (Spain) and it is ready to be used by any interested user by internet (https://emi-sstcdapp.unavarra.es/ICUsimulator). In order to access it, the username (ICU-simulator) and the password (ICU_S1mulat0r*) are required. When groups of physicians of different ICUs use this simulator, they will generate records of their decisions, which will enable us to test theories about physicians' decision-making, analyze triage processes and detect biases.

\section{DISCUSSION AND CONCLUSIONS}

Recent research shows that the use of MFS has a positive effect on the learning of participants. (Elsawah et al. 2017; Pennock et al. 2018). Based on this, we propose this ICU simulator as a learning tool from two different points of view.

On the one hand, this simulator could be used at universities by medicine and nursing students. This kind of tools has already been used successfully (Grunewald et al. 2003). In our case, students could run simulations by themselves, learning how ICUs are managed. They could take part in the decision-making process in a safe environment in which their decisions do not have bad consequences for patients. Not only that, but they could also see some simulations performed by ICU physicians, whose decisions are supposed to be the best. Therefore, students could compare how an expert decides about patients and how they do it themselves. Of course, physicians who work in ICUs could use the simulator in order to improve their knowledge of bed management.

On the other hand, from simulations performed by many physicians of the same hospital, it would be possible to learn not only individually but also in a collective way. For instance, comparing all files of decision-making we could detect which situations generate the most disparity between physicians. These scenarios could be categorized as conflicting scenarios because there is no unified response from physicians to manage this situation. In the same way, we could identify which patients are the most difficult to manage, because decisions about them are the most different (decision about admission, time to discharge...). All of this will allow physicians to generate conflictive scenarios (with the simulator) to test new policies in order to face these situations.

For now, a valid simulator has been built, which gathers the characteristics to reproduce the real operation of an ICU. The proposed methodology allows collecting relevant and objective information for the analysis of medical decisions, being able to generate scenarios that cause variability. The level of dissimilarity in decisions among physicians is analyzed in a quantitative way, besides identifying its causes and quantifying their influence on the management of ICU beds. Definitely, reducing the variability in the process of decision making about the discharge of a patient from the ICU could avoid early discharges and scheduled surgeries suspensions.

\section{ACKNOWLEDGMENTS}

This paper has been supported by grant MTM2016-77015-R (AEI, FEDER).

\section{A SET OF PATIENT VARIABLES}

- Informative patient's data: age, gender, weight, LoS, provenance, principal diagnostic, personal history and type of patient (Fixed values for each patient and considered outside the 275 variables).

- Neurological parameters (10): both pupil size in millimeters and reactivity, Glasgow Coma Scale (Glasgow-Motor response, Glasgow-Eye response and Glasgow-Verbal response), external ventricular drain and RASS scale.

- Hemodynamic parameters (17): oxygen saturation (\%), temperature in degrees Celsius $\left({ }^{\circ} \mathrm{C}\right)$, type of heart rhythm, heart rate $(\mathrm{rpm})$, systolic blood pressure $(\mathrm{mmHg})$, diastolic blood pressure $(\mathrm{mmHg})$ (invasive are non-invasive), mean arterial blood pressure $(\mathrm{mmHg})$, pacemaker rhythm $(\mathrm{y} / \mathrm{n})$, type of pacemaker, pacemaker operating modes, pacing rate (rpm), pacing capture threshold (mA), etc. 


\section{Garcia-Vicuña, Mallor, Esparza, and Mateo}

- Respiratory parameters (14): spontaneous breathing trials, tracheotomy (y/n), fenestrated cannula/speaking valve $(\mathrm{y} / \mathrm{n})$, tracheostomy cap $(\mathrm{y} / \mathrm{n})$, conventional mechanical ventilation $(\mathrm{y} / \mathrm{n})$, noninvasive positive pressure ventilation $(y / n)$, reservoir mask $(y / n)$, Venturi mask $(y / n)$, nasal cannula (L/minute), inspired gas flow with high-flow nasal cannula oxygen therapy (L/minute), fraction of inspired oxygen with high-flow nasal cannula oxygen therapy $(\%)$, fraction of inspired oxygen $(\%)$, extrinsic positive end-expiratory pressure $\left(\mathrm{cmH}_{2} \mathrm{O}\right)$ and respiratory rate (rpm).

- Kidney parameters (2): continuous renal replacement therapies $(\mathrm{y} / \mathrm{n})$ and intermittent hemodialysis $(\mathrm{y} / \mathrm{n})$.

- Balances (2): diuresis ( $\mathrm{mL})$ and chest drainage $(\mathrm{mL})$.

- Medication (123): plasmalyte $(\mathrm{mL} / \mathrm{h})$, propofol $2 \%(\mathrm{mg} / \mathrm{Kg} / \mathrm{h})$, midazolam $50 \mathrm{mg}(\mu \mathrm{g} / \mathrm{Kg} / \mathrm{h})$, remifentanil / ultiva $(\mu \mathrm{g} / \mathrm{Kg} / \mathrm{min})$, morphic chloride $(\mathrm{mg} / \mathrm{h})$, intravenous fentanyl $(\mu \mathrm{g} / \mathrm{Kg} / \mathrm{h})$, etc.

- Events and care (54): percutaneous tracheostomy, cardiopulmonary resuscitation, defibrillation, electrical cardioversion, pharmacologic cardioversion, transcutaneous pacing, pericardiocentesis, pulmonary embolectomy, etc.

- Infections (5): type of infection, origin, inflammatory response, germs and antibiotics.

- Analytics (37): hemoglobin (g/dL), hematocrit (\%), leukocytes (X10\%/L), neutrophils (\%), Lymphocytes (\%), monocytes (\%), eosinophils (\%), basophils (\%), etc.

- Gasometry (7): $\mathrm{pH}, \mathrm{pCO}_{2}(\mathrm{mmHg}), \mathrm{pO}_{2}(\mathrm{mmHg})$, lactate $(\mathrm{mmol} / \mathrm{L})$, saturation $(\%), \mathrm{HCO}_{3}$ $(\mathrm{mmol} / \mathrm{L})$ and base excess $(\mathrm{mmol} / \mathrm{L})$.

- $\quad$ Reports (4): admission, medical, nursing and clinical reports.

\section{REFERENCES}

Bai, J., A. Fügener, J. Schoenfelder, and J.O. Brunner. 2018. “Operations Research in Intensive Care Unit Management: a Literature Review". Health Care Management Science 21(1):1-24.

Barado, J., J. M. Guergue, L. Esparza, C. Azcarate, F. Mallor, and S. Ochoa. 2012. "A Mathematical Model for Simulating Daily Bed Occupancy in an Intensive Care Unit". Critical Care Medicine 40(4):1098-1104.

Bean, D. M., P. Taylor, and R. J. B. Dobson. 2019. "A Patient Flow Simulator for Healthcare Management Education”. BMJ Simulation and Technology Enhanced Learning 5(1):46-48.

Brailsford, S. and J. Vissers. 2011. "OR in Healthcare: A European Perspective". European Journal of Operational Research 212(2):223-234.

Cardoso, L. T., C. M. Grion, T. Matsuo, E. H. Anami, I. A. Kauss, L. Seko, and A. M. Bonametti. 2011. "Impact of Delayed Admission to Intensive Care Units on Mortality of Critically Ill Patients: a Cohort Study". Critical Care 15(1):R28.

Chalfin, D. B., S. Trzeciak, A. Likourezos, B. M. Baumann, R. P. Dellinger, and the DELAY-ED Study Group. 2007. "Impact of Delayed Transfer of Critically Ill Patients From the Emergency Department to the Intensive Care Unit". Critical Care Medicine 35(6): 1477-1483.

Chan, C. W., V. F. Farias, N. Bambos, and G. J. Escobar. 2012. "Optimizing Intensive Care Unit Discharge Decisions with Patient Readmissions". Operations Research 60(6):1323-1341.

Costa, A. X., S. A. Ridley, A. K. Shahani, P. R. Harper, V. De Senna, and M.S. Nielsen. 2003. "Mathematical Modelling and Simulation for Planning Critical Care Capacity". Anaesthesia 58(4):320-327.

Dobson, G., H. H. Lee, and E. Pinker. 2010. “A Model of ICU Bumping”. Operations Research 58(6):1564-1576.

Elsawah, S., A. McLucas, and J. Mazanov. 2017. "An Empirical Investigation Into the Learning Effects of Management Flight Simulators: a Mental Models Approach”. European Journal of Operational Research 259(1):262-272.

Frost, S., V. Tam, E. Alexandrou, L. Hunt, Y. Salamonson, P. Davidson, M. J. Parr, and K. M. Hillman. 2010. "Readmission to Intensive Care: Development of a Nomogram for Individualising Risk". Critical Care and Resuscitation 12(2):83-89.

Gantner, D., K. Farley, M. Bailey, S. Huckson, P. Hicks, and D. Pilcher. 2014 "Mortality Related to After-hours Discharge from Intensive Care in Australia and New Zealand, 2005-2012". Intensive Care Medicine 40(10):1528-1535.

Griffiths, J. D., V. Knight, and I. Komenda. 2013. "Bed Management in a Critical Care Unit". IMA Journal Management Mathematics 24(2):137-153.

Grunewald, M., R. A. Heckemann, H. Gebhard, M. Lell, and W. A. Bautz. 2003. "Compare Radiology: Creating an Interactive Web-Based Training Program for Radiology with Multimedia Authoring Software”. Academic Radiology 10(5):543-553.

Halpern, N. A. and S. M. Pastores. 2010. "Critical Care Medicine in the United States 2000-2005: an Analysis of Bed Numbers, Occupancy Rates, Payer Mix, and Costs”. Critical Care Medicine 38(1):65-71.

Kim, S. C., I. Horowitz, K. Young, and T. A. Buckley. 2000, "Flexible Bed Allocation and Performance in the Intensive Care Unit”. Journal of Operation Management 18(4):427-443. 
Lamé, G. and R. K. Simmons. 2018. "From Behavioural Simulation to Computer Models: How Simulation Can Be Used to Improve Healthcare Management and Policy”. BMJ Simulation and Technology Enhanced Learning Published Online First: 20 October 2018.

Mallor, F. and C. Azcárate. 2014. "Combining Optimization with Simulation to Obtain Credible Models for Intensive Care Units". Annals of Operations Research 221(1):255-271.

Mallor, F., C. Azcárate, and J. Barado. 2015. “Optimal Control of ICU Patient Discharge: From Theory to Implementation”. Health Care Management Science 18(3):234-250.

Mallor, F., C. Azcárate, and J. Barado. 2016. "Control Problems and Management Policies in Health Systems: Application to Intensive Care Units". Flexible Services and Manufacturing Journal 28(1-2):62-89.

Moxnes, E. and A. K. Saysel. 2009. "Misperceptions of Global Climate Change: Information Policies". Climatic Change 93(1):1537.

Ouanes, I., C. Schwebel, A. Francais, C. Bruel, F. Philippart, A. Vesin, L. Soufir, C. Adrie, M. Garrouste-Orgeas, J. F. Timsit, B. Misset, and Outcomerea Study Group. 2012. "A Model to Predict Short-Term Death or Readmission After Intensive Care Unit Discharge”. Journal of Critical Care 27(4):422-429.

Pennock, M. J., Z. Yu, K. B. Hirschman, K. Pepe, M. V. Pauly, M. D. Naylor, and W. B. Rouse. 2018. "Developing a Policy Flight Simulator to Facilitate the Adoption of an Evidence-Based Intervention". IEEE Journal of Translational Engineering in Health and Medicine 6:1-12.

Rauner, M. S., H. Niessner, U. Leopold-Wildburger, and N. Perić. 2016. “A Policy Management Game for Mass Casualty Incidents: An Experimental Study”. Flexible Services and Manufacturing Journal 28(1-2):336-365.

Ridge, J. C., S. K. Jones, M. S. Nielsen, and A. K. Shahani. 1998. "Capacity Planning for Intensive Care Units”. European Journal of Operational Research 105(2):346-355.

Rodriguez-Carvajal, M., D. Mora, A. Doblas, M. Garcia, P. Dominguez, A. Tristancho, and M. Herrera. 2011. "Impacto de las Altas No Programadas en la Mortalidad Hospitalaria Tras la Estancia en una Unidad de Cuidados Intensivos". Medicina Intensiva 35(3):143-149.

Shmueli, A., C. L. Sprung, and E. H. Kaplan. 2003 “Optimizing Admissions to an Intensive Care Unit”. Health Care Management Science 6(3):131-136.

Sterman, J. D. 1989. "Misperceptions of Feedback in Dynamic Decision Making”. Organizational Behavior and Human Decision Processes 43(3):301-335.

Teres, D. 1993. "Civilian Triage in the Intensive Care Unit: the Ritual of the Last Bed”. Critical Care Medicine 21(4):598-606.

\section{AUTHOR BIOGRAPHIES}

DANIEL GARCIA-VICUÑA studied industrial engineering at the Public University of Navarre, Spain. Currently, he is a Ph.D. student at the Institute of Smart Cities of the Public University of Navarre. His research interests lie in the field of complex real problems simulation modelling. His email address is daniel.garciadevicuna@unavarra.es.

FERMIN MALLOR is Full Professor of Statistics and Operations Research at the Public University of Navarre, Spain. He holds a Ph.D. and MSc in Mathematics. He has been visiting researcher at the Missouri Science and Technology University, among others. His research interests include applications of simulation-optimization (classical and metaheuristics methods) in health, energy, logistics, and production. His email address is mallor@unavarra.es.

LAIDA ESPARZA is a physician at the Department of Intensive Care Medicine, Hospital Complex of Navarra, Spain. Currently, she is a Ph.D. student at the Department of Statistics and Operations Research at the Public University of Navarre. Her research interests lie in the field of simulation modelling and in the study of clinical decision-making processes and bed management in intensive care unit settings. Her email address is lesparza@navarra.es.

PEDRO MATEO received its Ph.D. degree in Sciences from the University of Zaragoza, Spain, in 1995. Since 1998 he is an associate professor in the Department of Statistical Methods of the University of Zaragoza, Spain. His current research interests include machine learning, multi-objective evolutionary algorithms, and simulation modelling. His email address is mateo@unizar.es. 